\title{
Smart Industry Monitoring and Controlling System Using IoT
}

\author{
Elumalai. $\mathrm{G}^{\mathrm{a}, 1}$, Nallavan. $\mathrm{G}^{\mathrm{b}}$ and Ramakrishnan. $\mathrm{R}^{\mathrm{c}}$ \\ ${ }^{a}$ Assistant Professor, Dept of ECE, Panimalar Engineering College, Chennai, India \\ ${ }^{\mathrm{b}}$ Associate Professor, Dept of ECE, TNPESU, Chennai, India \\ 'Professor, Dept of ECE, TNPESU, Chennai, India
}

\begin{abstract}
Air pollution in an ecosystem has proliferated industrial automation. This dissertation concentrates more on industrial automation and has design an embedded device with sensors to monitor and control the toxic gases in industries. This entire prototype is an excellent result for observing the toxic gases in industry and generates information by using data acquisition and transmission of data."Internet of Things (IoT)" is a important technology behind this and it provide platform to bring together all the devices in the world to the internet. In this dissertation, the parameters monitored are temperature, humidity and gas leakages in industries. The sensor senses the parameters and uploads these data to the cloud with the help of NodeMCU. If observed gas level is above the threshold which is the safety limit of operation, the first alert is intimated from the Google cloud and the controlling action carried out (ie) automatically close gas leakage valves and then industry will take immediate step to control pollution. Or else, the second alert message is sent through Electronic mail (e-mail) to restore the safe limit, as government play role to power outage in the industries. Cloud is used to store the sensed data, which is then transmitted and processed.
\end{abstract}

Keywords. Air pollution, Toxic gases, ECO-system, ESP8266 and IoT.

\section{Introduction}

In the recent years wireless technology and IoT grasped the most industrial area especially automation and control has increasing for need of upholding various sectors. Healthcare has prime importance in our day to day life. This paper reviewed about new industrialization with ESP8266 and arduino UNO. Indoor Air Quality (IAQ) is highly worsens industrial environments, which then spreads from indoor to outdoor, creating a large scale effect around the industrial areas. Long term and short term effects caused by Air pollution causes the people to concern about the air they breathe. The effect of air pollution from industry is monitored scarcely. Our aim is to monitor the air pollution from the heavy industry which leads to undesirable effects on the health of human beings and also affects the environment. Pollution level in comparison to the ambient air quality standards can be done by using monitoring. To protect the people against extreme air pollution. Robust monitoring systems are necessary to alert people and initiate actions. 


\section{Review of Related Literature}

Kavitha.B.C et al. (2019), insists on using intelligent sensors for pollution monitoring. Collected data from the sensors are sent to the Google cloud makes it possible to monitor the air pollution from anywhere in the world. In case of threating levels of air pollution, alerting is used. This is used in industry and the pollution by vehicular emissions. Rajalakshmi.R et al. (2019), Observes the toxic gases present in the air to ensure the safety of the people in that environment and make it available at any place in the world for monitoring. The composition of chemicals in air like carbon monoxide, LPG, methane and flammable gases is monitored using sensors and this data is sent to the cloud server, which is then represented pictorially for better understanding of the statistics. Rupali et al. (2018), cares for home and industrial safety using fire and gas detection systems. This system detects the leakage of gas and fire using sensing circuit, which is then controlled by microcontroller which in turns triggers the alarm system to alert the leakage of gas and fire. Using GSM modems, SMS are being sent to notify the user. In addition, it is designed with mechanism to sprinkle the water using water sprinkler when there is a fire or gas leakage. MQ-6 and MQ-9 used as gas sensors to detect the gas leakage. IR flame sensor is used for fire detection, which detects the fire and notifies the user using SMS. Manish Verma et al. (2018), uses microcontroller based system to investigate about the toxic gas detection and alerting system. LCD display is used where the levels of hazardous gases like LPG and propane was displayed each second. Authorized person is notified with email and also using alarm generation mechanism. This automated detection and alerting mechanism helps to resolve the problem as soon as possible. Angelica Nieto Lee et al. (2018), this paper focuses on integrating all the contextual data, to provide accurate and relevant information as per the need. System information that already exists but has not been integrated into the monitoring system like 3D models and manuals. It is context aware industrial monitoring systems, which provide information based on system state, environmental conditions and functionalities of the devices in that environment. Ishwarya et al. (2018), insisted on automation of many small tasks around us using Internet of Things (IoT) in order to improve the quality of living. IoT is used for enhancing existing safety standards, using automation process. Gas leakages in open or closed areas can prove to be dangerous and fatal. Traditional gas leakage systems can able to detect the leakage but cannot able to alert the user. Alerting System can be established to alert the authorized person and to perform the data analytics from the obtained readings.

\section{Proposed System}

The figure 1 below shows the block diagram and prototype of the proposed system. The components used to design the hardware are Arduino UNO, Semiconductor sensor (MQ6 \& MQ7), ESP8266, Relay, Power supply. MCP3008 is an analog to digital convertor. Analog values from the sensors are given to MCP3008, which is an 8 channel ADC, that converts the analog data to digital data which is then sent to NodeMCU. The parameters are monitored using DHT11, MQ-6, and MQ-7 sensors. The sensor senses their parameters regarding the temperature, humidity and gas level and uploads these data to the cloud with the help of WiFi device (NodeMCU). If the level of the gas reaches above the normal level, the first alert is intimated from the 
Google cloud as it is automatically closes gas leakage valve and then industry will take immediate step to control pollution. Or else, the second alert message is sent through Short Message Service (SMS) to restore the safe limit intended so and as government play role power outage in the industries.

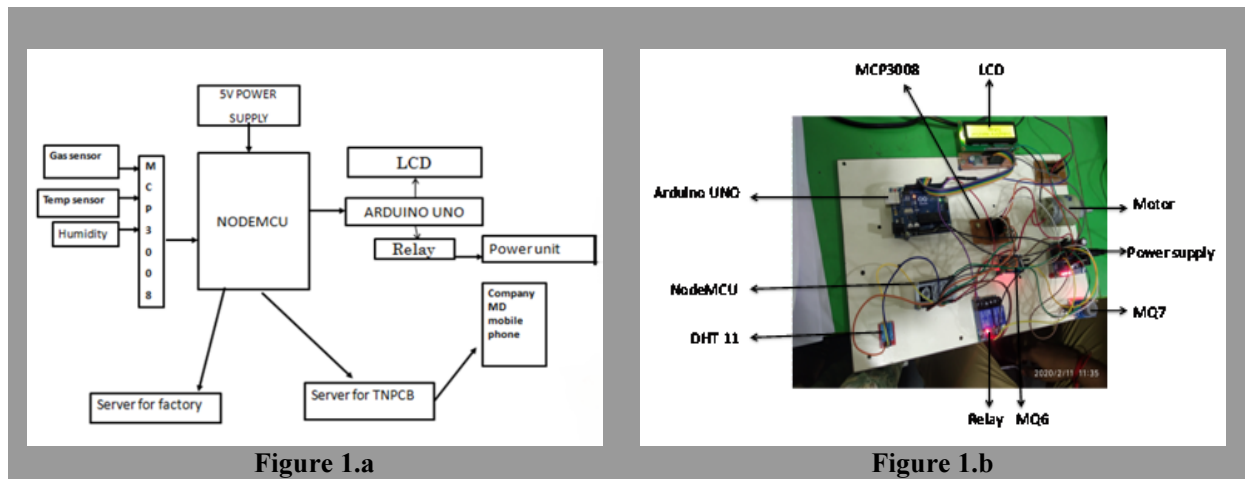

Figure 1. Block diagram and prototype of the proposed system

\section{Result and Discussion}

We are going to test our prototype for different cases are discussed below. For our analysis Gas-1 represents carbon monoxide (CO), Gas-2 represents Isobutane, Propane, Liquefied Natural Gas (LNG) and Methane. Temp represents temperature. Humi represents humidity. Status represents either normal or emergency based on the industrial gas leakage level.

\section{Analysis for Gas-1}

In the figure 2.a,the permissible level of Gas-1 is below 300. In this case, Gas-1 does not reaches permissible level, so it is not harmful to the environment. Hence the status is normal.In the figure 2.b, the permissible level of Gas-1 is above 300. In this case, Gas-1 reaches above permissible level so it is harmful to the environment. Hence the status is emergency.

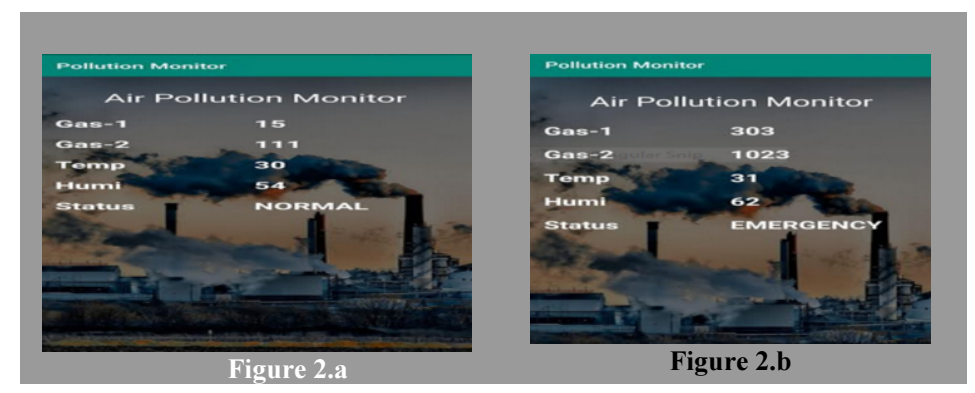

Figure 2. Output of Gas-1 
Analysis for Gas-2

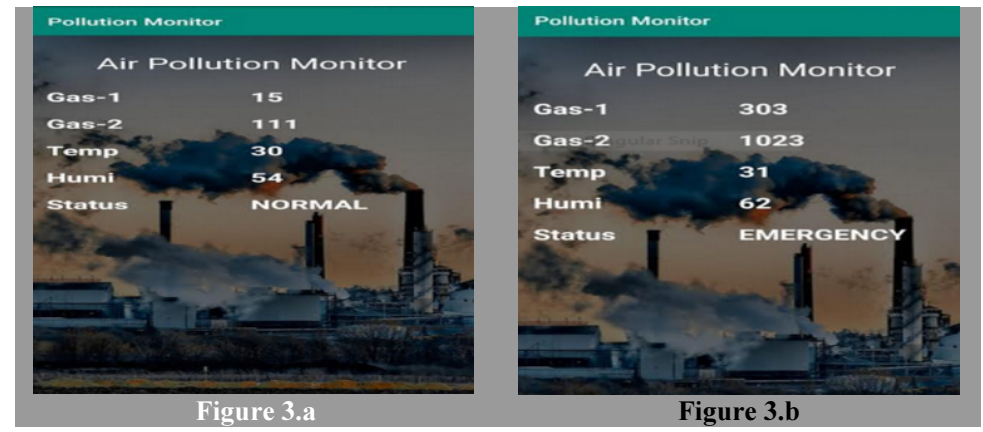

Figure 3. Output Gas-2

In the figure 3.a, the permissible level of Gas-2 is below 300. In this case, Gas-2 does not reaches above permissible level so it is not harmful to the environment. Hence the status is normal. In the figure 3.b, the permissible level of Gas-2 is above 300. In this case, Gas-2 reaches above permissible level so it is harmful to the environment. Hence the status is emergency.

\section{Conclusion}

In this paper smart Industry Monitoring system based on IoT is proposed which can effectively monitor and controls with alert. A prototype based on Arduino UNO was developed which could sense the concentration of gases. The real time data information obtained from the different sensors has been uploaded to Google Cloud which displayed in the LCD. In addition to this other parameters like temperature, humidity was measured. Provision was also made to vigilant the workers in case of any emergency. The system provides consistently and accurate analysis to prevent any case of accidents. This system makes use of Arduino UNO providing cheap solutions for safety. Slight modification of the model enables the user to adapt it to any environment. Predictive maintenance is an upcoming industrial need, for which the proposed model can be improvised. In case of gas leakage the concentration of gas varies from point to point which has to be analyzed. Moreover, the gases diffusing out during leakage may also combine among themselves producing other by products which have to be dealt in detail.

\section{Future Scope}

This prototype helps the industrial site from gas leakage deduction and faster resolution of problems afforded by a higher level of expertise focused on control system. This methodology could be applied to monitor distribution network of natural gas as well as industrial, commercial, residential gas pipelines in order to provide a safe operation and to avoid severe human health injuries caused by gas leakages. Proposed solution can act as a automatic vehicle health feed for manufacturer to improve their quality by providing regular vehicle services. 


\section{References}

[1] Kavitha.B.C, Vallikannu.R . IoT based intelligent industry monitoring system. International Conference of Signal Processing and Integrated Networks, 2019

[2] Rajalakshmi.R, Vidhya.J .Toxic environment monitoring using sensors based on IoT. International Journal of Recent Technology and Engineering, 2019.

[3] Rupali, S.Gajare, P.Mahajan .Home and industrial safety system for fire and gas leakage detection. International Research Journal of Engineering and Technology, 2018.

[4] PathRastogi, DikshaTripathi .Gas leakage detection and prevention kit provision with IoT. International Research Journal of Engineering and Technology, 2018.

[5] IshwaryaGawali, V.Nayakwadi, PriyankaRajmanes .Gas leakage detection and smart alerting system using IoT. International Journal of Innovative Research in Science, 2018.

[6] V.Goulao, R Paulo, J Salvado, G Martins, A Granjeia, A new monitoring and data logger system for industrial cooling equipment application, Institute Of Electrical And Electronics Engineers , 2017.

[7] Mohammed Y Aalsalem, NasrullahArmi .Intelligent oil and gas well monitoring system based on IoT .Institute Of Electrical And Electronics Engineers. 2017.

[8] KanappanA,Hariprasad.K ,Toxic gas and radiation detection monitoring using IoT, International Journal of Engineering Resesarch and Technology, 2017.

[9] Fabien Charaim, Yusuf BugraErol, Kris Pister. Wireless gas leakage detection and localization Institute Of Electrical And Electronics Engineers, 2016.

[10] KanZheng, Shaohang Zhao, Zhe Yang, Xiongxiong, Design and implementation of LPWA-based air quality monitoring system, Institute Of Electrical and Electronics Engineers, 2016.

[11] NavreentinderKaur, Rita Mahajan, Deepak Bagai, Air quality monitoring system based on Arduino microcontroller, International Journal of Innovative Research in Science, Engineering and Technology, 2016.

[12] OnengiyeM.Georgewill, Ezeofor, Chukwunazo .Design and implementation of SMS based industrial/home gas leakage monitoring and detection alarm system .International Journal of Engineering Trends and Technology, 2016. 